\title{
Dictionary-based Filling of the Missing Wedge in Electron Tomography.
}

\author{
Patrick Trampert $^{1,2}$, Delei Chen ${ }^{3}$, Sviatoslav Bogachev ${ }^{1,2}$, Tim Dahmen ${ }^{1,2}$ and Philipp Slusallek ${ }^{1,2}$ \\ 1. German Research Center for Artificial Intelligence GmbH (DFKI), 66123 Saarbrücken, Germany. \\ 2. Saarland University, 66123 Saarbrücken, Germany. \\ 3. The Institute of Nanoscopy, Maastricht University, 6211 LK Maastricht, The Netherlands.
}

A new method for dealing with electron tomography data from incomplete projection sets is proposed. The approach is inspired by exemplar-based inpainting techniques in image processing [1] and heuristically generates data for missing projection directions. The method has also been extended to work on three dimensional data. In general, electron tomography reconstructions suffer from elongation artifacts along the beam direction at $0^{\circ}$ tilt. These artifacts can be seen in the corresponding Fourier domain as a missing wedge. The new method synthetically generates projections for these missing directions with the help of a dictionary-based approach that is able to convey both structure and texture at the same time. It constitutes a preprocessing step that can be combined with any tomographic reconstruction algorithm. The new algorithm was applied to the well-known Shepp-Logan phantom. Visually, the synthetic projections, reconstructions, and corresponding Fourier power spectra showed a decrease of the typical missing wedge artifacts. Quantitatively, the inpainting method is capable to reduce missing wedge artifacts and improves tomogram quality with respect to several figures-of-merit.

The new algorithm was implemented with the software package Ettention [2] and applied to the datasets with fixed parameters. We assessed the capabilities of the method with several evaluation metrics, namely root mean square error (RMSE), Peak signal-to-noise ratio (PSNR), and the structural similarity index (SSIM). The generated projections (Figure 1) look very similar to the ground truth (GT) projections. Beginning from $-63^{\circ}$ (to $-90^{\circ}$ ) as first inpainted projection there is nearly no difference recognizable between inpainted and real projection. The further away the inpainted projections are from the input of range $-62^{\circ}$ to $62^{\circ}$, the more the inpainted projections deviate from the real ones. Nevertheless, even the projection at $-90^{\circ}$ is clearly identifiable as a Shepp-Logan phantom projection. Projections in the upper tilt angle range between $63^{\circ}$ and $90^{\circ}$ behave analogously. The evaluation measures also showed the impact of the method. RMSE between GT and inpainted projections was 0.04, PSNR 28.08, and SSIM 0.87, which shows that the new method generates meaningful projections.

Reconstructions were performed with [2] using simultaneous iterative reconstruction technique (SIRT) with 300 iterations. As can be seen in Figure 2, the reconstruction with incomplete data has clearly visible artifacts and the contours are not closed. In contrast, the reconstruction with the inpainted dataset has closed contours and the missing wedge artifacts are not visible any more. In the interior there are some smearing artifacts compared to GT, which are not severe. Visual results are supported by the evaluation measures. RMSE between incomplete data and GT was 0.08 and between inpainted data and GT 0.01, PSNR 22.2 compared to 37.8, and SSIM 0.56 compared to 0.84 , which shows that reconstructions of data generated with the new method are superior to missing wedge data reconstructions.

Performance issues are the main concern of the new method, but we are quite optimistic that this can be fixed by technical improvements. The current runtime for a dataset with a resolution $256 \times 256$ is around 90 minutes when generating 60 projections. Despite that fact, experiments showed, that exemplar-based 
inpainting is capable of generating meaningful projections where data is missing due to limited tilt range in data acquisition leading to reduced missing wedge artifacts in subsequent reconstructions. [3]

\section{References:}

[1] A Criminisi, "Region filling and object removal by exemplar-based image inpainting", IEEE Trans. Nucl. Sci., 2004, vol. 3: 21-43.

[2] T Dahmen et al, “The Ettention Software Package”, Ultramicroscopy, 2016 Feb, vol. 161: 110-118.

[3] Research has been funded within the European Research Project NOTOX (FP7-267038). The authors thank the DFKI GmbH for additional funding and for providing the necessary infrastructure.

a)

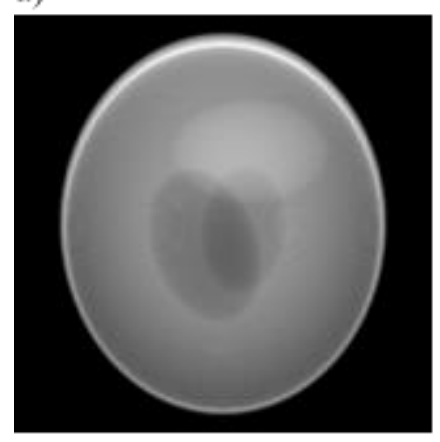

e)

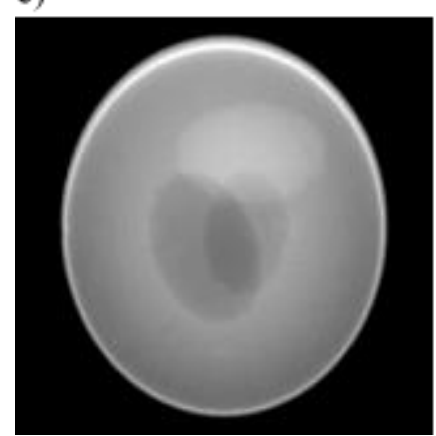

b)

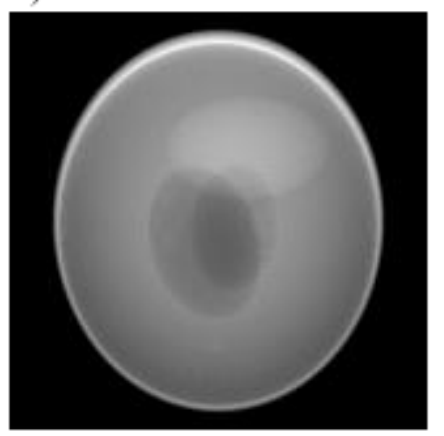

f)

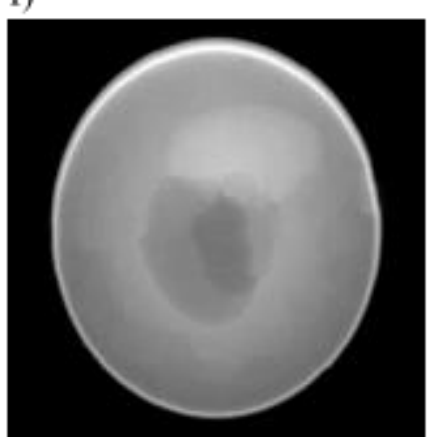

c)

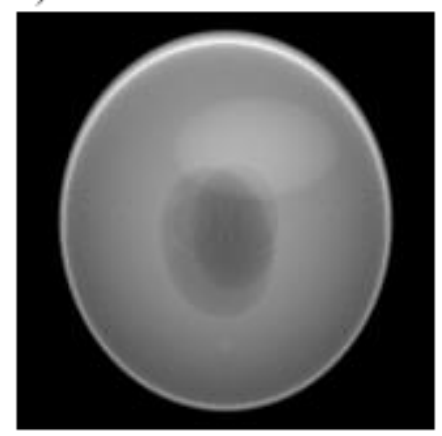

g)

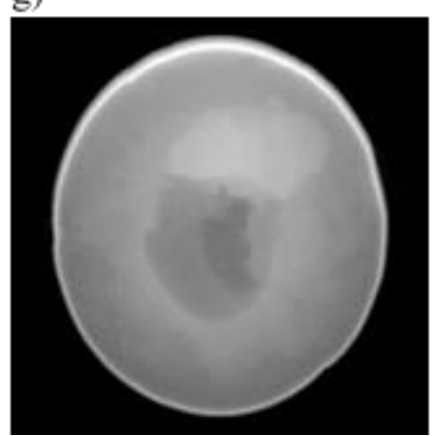

d)

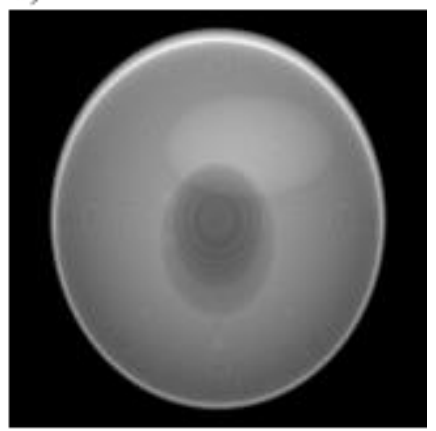

h)

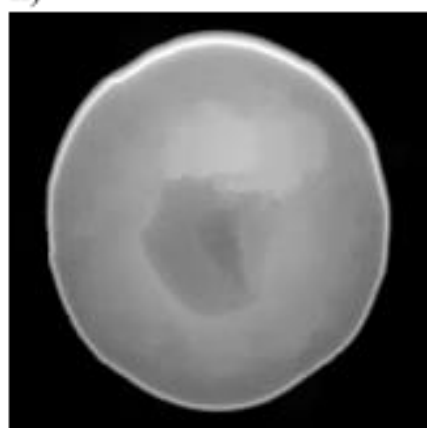

Figure 1. Comparison of several projections between ground truth data (upper row) and inpainted data (lower row) of Shepp-Logan phantom. From left to right projections with tilt angle $-63^{\circ},-70^{\circ},-78^{\circ}$, and $-90^{\circ}$ are depicted.

a)

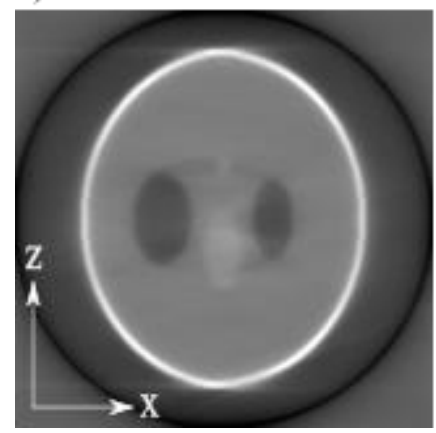

b)

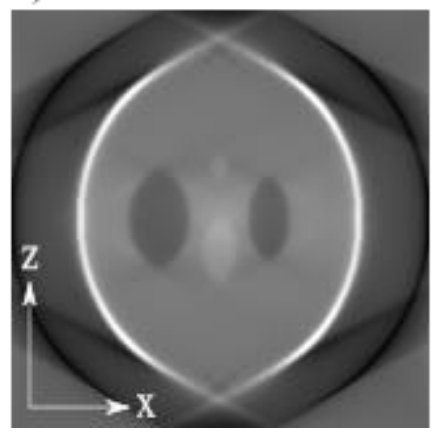

c)

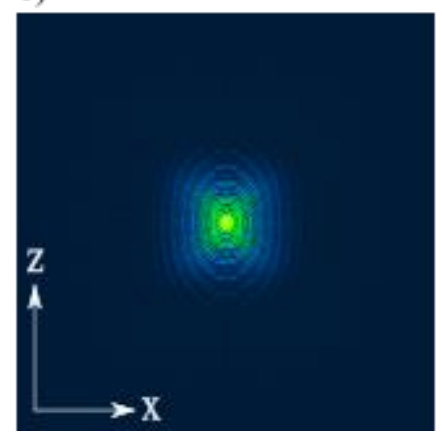

d)

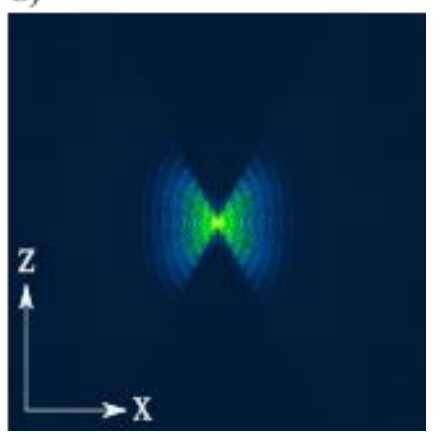

Figure 2. Comparison of reconstruction results of the Shepp-Logan phantom. a) reconstruction of inpainted dataset, b) reconstruction of incomplete dataset with projections from $-62^{\circ}$ to $62^{\circ}$, c) Fourier spectrum of inpainted reconstruction, d) Fourier spectrum of incomplete data reconstruction. 